\title{
Estimating costs of post-abortion services. General Hospital Aurelia Valdivieso, Oaxaca, Mexico
}

Population Council

Follow this and additional works at: https://knowledgecommons.popcouncil.org/departments_sbsr-rh

Part of the Demography, Population, and Ecology Commons, International Public Health Commons, Quality Improvement Commons, and the Women's Health Commons How does access to this work benefit you? Let us know!

\section{Recommended Citation}

"Estimating costs of post-abortion services. General Hospital Aurelia Valdivieso, Oaxaca, Mexico," INOPAL III Final Report. Oaxaca, Mexico: Population Council, 1999. 


\section{ESTIMATING COSTS OF POST-ABORTION SERVICES. GENERAL HOSPITAL AURELIO VALDIVIESO, OAXACA. MEXICO.}

FINAL REPORT. INOPAL III. 


\section{INTRODUCTION}

Health care systems around the world are facing the problems of providing quality care with increasingly constrained resources. While modern practices and diagnostic tests have made pregnancy a much less risky event than in the past, not all pregnancies have a favorable outcome. Often times the pregnancy does not reach full term and a woman aborts the fetus spontaneously. Spontaneous abortion is a natural though unintended event that occurs in $15 \%$ to $45 \%$ of all known pregnancies. Abnormal products of conception are the overwhelming cause of spontaneous abortion, also known as miscarriage. Furthermore, the earlier the gestation, the more likely is abortion. It is estimated that $75 \%$ of spontaneous abortions occur before 16 weeks of gestation - in some cases, before the woman was even aware that she was pregnant - and approximately $60 \%$ occur before 12 weeks of gestation (Benson and Pernoll, 1994).

One of the major causes of maternal mortality worldwide is unsafe abortion. Approximately 200,000 women in developing countries die each year from complications of induced abortions, from an estimated 40-60 million induced abortions yearly worldwide (Germain, 1989). Some health care systems spend close to $60 \%$ of their obstetrics/gynecology budgets treating this problem (von Allmen, 1977). The standard of care requires that unsafe abortion be treated in a hospital/medical facility with adequately trained personnel. Studies done at Kenyatta National Hospital in Nairobi, show that 25\% of all obstetric/gynecology hospital admissions were for cases of incomplete abortion (Agarwal et al., 1982). Given the high level of resources devoted to treating this condition, it is imperative to research and develop costeffective methodologies to provide quality care.

Unsafe abortion performed by untrained and inexperienced providers and its resulting 
complications is the fourth leading cause of maternal mortality in Mexico (Secretaria de Salud, Mexico, 1992). The Alan Guttmacher Institute calculated that at the beginning of the decade, there were over half a million cases of induced abortion per year in Mexico alone. Many authorities feel that these statistics underestimate the true picture, as many abortion-related deaths are attributed to non-specific hemorrhage or infection.

Similar to the case in other developing countries, the costs associated with incomplete abortion in Mexico are extremely high, and treatment for this condition consumes the greatest amount of reproductive health care resources after normal births. In addition, women often harbor a psychological burden of anxiety and guilt that is seldom measured. For these reasons, incomplete and unsafe abortion constitutes a serious public health problem in Mexico. Strengthening the capacity of health care institutions to provide high quality post-abortion care services that are cost-effective, accessible and sustainable is a major public health objective.

Evacuation by dilatation and curettage $(D \& C)$ is still the standard of care for managing incomplete abortion in many developing countries (McLaurin et al., 1991). Efforts to control costs and improve quality of care have led to the development of the manual vacuum aspiration (MVA) technique as an advancement over the traditional D\&C method. Research has shown that MVA is more cost-effective than D\&C. Work to this effect has been undertaken in Kenya, Colombia and Mexico (Magotti et al., 1995; Johnson et al., 1993; Careres et al, 1981). The common finding of these studies were that outpatient management using either MVA or D\&C resulted in shorter patient stays, and hence lesser resource utilization, leading to decreased overall systemwide costs. It is important to note that these studies did not overtly address the issue of quality of care received by patients undergoing either D\&C or the MVA procedure. 
To improve its service delivery model, the Aurelio Valdivieso General Hospital in Oaxaca, with support from the Reproductive Health Program of the Population Council and the European Union, implemented a redesigned service model for women seeking care for incomplete abortion. Prior to the implementation of the service model, the hospital predominantly used the $\mathrm{D} \& \mathrm{C}$ technique and did not routinely offer family planning counseling and/or contraceptive education to the patients.

The goal of the service model was to provide high quality care in a cost-efficient manner for cases of incomplete abortions. Integral components of this service model included:

- preferential use of Manual Vacuum Aspiration (MVA)

- provision of family planning counseling

- easy availability of contraceptive methods

- availability of patient educational material focused on feminine hygiene, contraception and post-abortion care.

The service model was developed in close consultation with various service providers and was preceded by meetings, seminars and discussion groups so that all obstetric/gynecology and hospital personnel were fully conversant with it. The improved service model was expected to improve quality of care and general patient satisfaction, leading to increased post-abortion contraceptive use, with decreased expenditures.

This study assesses the cost and quality implications of this new service model, with the explicit aim of quantifying the savings (if any) resulting from the use of the MVA procedure. This study is unique because it is an attempt to understand the complete costs of a post-abortion service model, identifying areas where maximal gains accrued, simultaneously addressing the 
issue of patient care quality.

\section{METHODS}

\section{STUDY SITE}

The Aurelio Valdivieso General Hospital, situated in the capital city of Oaxaca, is the largest public hospital in the state of Oaxaca, one of the poorest and most rural states located in the south of Mexico. This civil hospital is managed by the Ministry of Health and serves the general public, the majority of whom come from poor socioeconomic backgrounds. ${ }^{1}$ It also serves as a teaching hospital for the local medical school. Most patients come either from the surrounding urban areas or are referred from health centers in the largely indigenous, rural areas of the Oaxaca valley. In many cases, women must travel between three and eight hours to reach the hospital; approximately four women arrive each day to seek emergency treatment for incomplete or complicated abortions. Demand for services has grown dramatically in the last few years.

In February 1996 Valdivieso Hospital initiated an improvement process of its postabortion service with advice and support from the Population Council and the European Union. Prior to the intervention, women who arrived at the hospital emergency ward were usually treated with sharp curettage $(D \& C)$ under general anesthesia as the technique for uterine evacuation, and discharged within 48 hours. Family planning counseling was rare and contraceptive methods were practically unavailable for the majority of post-abortion patients

\footnotetext{
${ }^{1}$ In Mexico, almost one-third of the population has no insurance (private or social insurance) for medical care and thus receive care in public institutions similar to the Valdivieso Hospital.
} 
upon hospital discharge.

\section{TECHNIQUE OF UTERINE EVACUATION \& SERVICE MODEL}

\section{Routine hospital care prior to the intervention}

Patients usually presented to the emergency room and were evaluated by the resident physician on call. The waiting time to be examined depended on the severity of a woman's condition; in general, deliveries were given a higher priority than post-abortion cases.

During the initial medical exam, there was no privacy for the patient as the front of the small consulting area was protected by only a thin curtain and the back of the room was open, allowing visual access to hospital personnel passing by. The attending resident took the medical history, examined the patient, and recorded the observations in the clinical register. There was little interaction with the patient during this routine questioning, and often the physician addressed the patient only to request that she undress and lie on the examining table, with only a minimum explanation of her medical situation.

Patients were then admitted and assigned to one of eight beds in a room in the OB/GYN ward. Post-abortion patients were supposed to be located with women requiring hysterectomies or tubal ligations, but due to a shortage of space, they often roomed with new mothers and their babies. Because the Dr. Aurelio Valdivieso General Hospital serves as a teaching hospital, the medical residents and interns are generally responsible for the patient's diagnosis, surgical treatment and monitoring. Interns were usually responsible for taking the patient's medical history, although this vital step would often occur after the uterine evacuation procedure had been carried out. The surgical procedure (D\&C) was carried out in the surgical suite under 
general anaesthesia.

During the post-operative recovery period, nurses would monitor the patient's vital signs, but there was little further interaction. Nurses did not counsel the patients about post-procedure precautions or contraceptive method use. Prior to discharge, the nurse would instruct the patient in a perfunctionary manner about diet, medications and follow up appointments.

\section{Refined Service Delivery Model}

The objective of the service delivery model was to improve the post-abortion quality of care while conserving resources. This was done by: 1) modifying hospital procedures to reduce waiting time, to improve pain management and to ensure patient privacy; 2) using the MVA technique when indicated (instead of D\&C); 3) providing post-abortion contraceptive counseling and providing educational material and contraceptives to patients.

Modification of hospital procedures : As a result of workshops held with the gynecological service staff, hospital physicians and administrators, an improved protocol for treatment and care of the incomplete abortion patient was implemented. All patients with a fundal height less than $12 \mathrm{~cm}$ would undergo uterine evacuation using manual vacuum aspiration. All surgical operating rooms would function 24 hours a day, seven days a week. This would enable post-abortion patients arriving at night or during weekends to undergo MVA without undue delay. Family planning counseling would become a mandatory component of post-abortion care services, and the family planning service was to be well stocked with hormonal contraceptives, condoms, and IUDs, so as to supply a broader range of options for the users. Contraceptives were to be given to the patient prior to hospital discharge. 
A door was installed in the consulting room of the emergency ward to replace the curtain. In addition, the back door to the consulting room was sealed off to provide greater privacy.

Improvement of patient treatment, information and counseling: Workshops were conducted to (a) sensitize providers to the realities of women who experience an abortion, with the intention of modifying their often negative attitudes towards these women and (b) to educate physicians and nurses in the provision of post-abortion counseling including family planning information.

These sessions emphasized that all women be provided information during the postabortion period about their almost immediate return to fertility and the possibility and risks of getting pregnant before their next menstrual period; safe methods to avoid or postpone pregnancy for at least six months; and where to receive family planning services and methods on a regular basis. Furthermore, providers were instructed in supporting a woman's decision to use a contraceptive method, helping identify which method was best suited for the patient, and training her (and her partner, as the case may be) to use the method correctly. It was repeatedly emphasized that the patient be given the opportunity to ask questions and express her concerns.

In order to reinforce the points made during discussions and workshops, posters encapsulating the salient points were posted throughout the hospital. Patient brochures were printed and made available in waiting and consulting rooms. 


\section{DATA COLLECTION AND ANALYSES}

\section{Cost Analyses}

Cost analyses was based on the approach developed by IPAS (Abernathy et al, 1993). Fixed direct costs were estimated from information provided by hospital administrators. These officials furnished information on the salaries and average costs of hospital resource use.

Variable costs were estimated by closely following individual cases of incomplete or complicated abortion from the time of admission to hospital discharge ("shadow observation"). These were patients with incomplete abortion, abortion in progress or a case of dead and retained fetus, who were admitted to the gynecology floor through the emergency ward. Cases of dysfunctional bleeding, threatened abortion, and abortions with complications such as uterine perforations and sepsis, and cases that were referred to the hospital from medical offices or other referral sources were excluded. ${ }^{2}$ To ensure these observations captured normal variations in staffing and caseload, these observations were conducted over a minimum ten-day period. Trained nurses using checklists noted the material resources (medical instruments, drugs, disposable supplies, etc.) used; time data were noted by observing time spent by patients in each department or section and time devoted by hospital personnel in providing direct patient care. Indirect costs were not included in the analyses. ${ }^{3}$

\footnotetext{
${ }^{2}$ These patients were excluded because these are not the "typical" patients seen by the hospital on a routine basis; these few cases would have biased the mean costs greatly in their direction. Also, the resources used to treat complications would have been nearly identical irrespective of the evacuation procedure used (D\&C or MVA).

${ }^{3}$ Indirect costs include the depreciation of infrastructure and capital goods such as vehicles, medical and non-medical equipment, instruments, etc., and state and central level administrative costs. These were not included because there is no reliable accounting information available at the central or state levels to ascertain these costs and also because from a policy perspective, these are outside the control of hospital directors and administrators. Moreover, they would have been, in all probability, equal in the pre- and post-intervention period.
} 
Eleven cases were observed in this manner during a 20 day period in January 1996 to estimate pre-intervention costs. Post-intervention costs were estimated by shadow observing twenty-five patients in July 1997. Costs were noted in Mexican pesos and in order to adjust for inflation, all costs are reported in constant January 1997 US dollars. ${ }^{4}$ Cost categories reported in the analyses are listed below.

Hospitalization: Total yearly hospital expenditures for 1995 and 1996 were abstracted from the hospital's accounting system to ascertain hospitalization costs. Budget items included salaries of medical and administrative staff, medical supplies, instruments and services, food services, maintenance and services and general services. These costs were pro-rated and adjusted for censable beds and occupancy rate, to estimate the cost per bed/day of use.

Staff: These costs were estimated by pro-rating average salaries and benefits for the time spent (in minutes) by specific providers in providing direct care to the patient. Time spent by specific providers (physicians, nurses, and administrative/support staff) at each station was explicitly noted in the shadow observations. Time of personnel attention refers to the time spent by the provider in direct care of the patient. For analytical purposes, this category excludes costs of family planning counseling, which is considered an intervention cost.

Instruments and supplies: Specific quantities of each resource consumed were noted in the shadow observations. Consumable resources noted included medical instruments, equipment and supplies, medicines, sterilization materials and contraceptives. Costs of consumables are calculated on the basis of unit purchase price for the hospital. Transportation costs are included,

\footnotetext{
${ }^{4}$ Inflation in Mexico was 52\% in 1995, 27\% in 1996 and 8.7\% during the first trimester of 1997.
} 
but storage costs are excluded in this calculation. ${ }^{5}$

Family planning services: Since provision of family planning services was an important element of the service delivery model, its cost was estimated separately. Observers registered the times of initiation and termination of all family planning information or counseling, and noted the number and type of contraceptives provided to each patient. Costs were then estimated by pro-rating the average salary and benefits of the provider (physician or nurse) for the time spent per patient, and determining the purchase price of the contraceptive from the hospital administration records.

Intervention costs: It is vital for policy makers to know beforehand what a proposed intervention is going to cost, so that these can be included in a cost-benefit analyses of the proposed intervention. Keeping this in mind, we estimated the start-up costs for the service delivery model separately. Intervention costs included direct project costs (project management, costs of training sessions, travel costs, etc.) supplies provided by IPAS (equipment including MVA syringes, printed materials, etc.), and hospital costs (cost of personnel time for training, follow up meetings, supervision and monitoring). These intervention start up costs were be amortized over time.

Quality of Care

In addition to the shadow observations to estimate costs (vide supra), surveys were conducted to assess the service model's impact on quality of care. A baseline (pre-intervention)

\footnotetext{
${ }^{5}$ Storage costs are subsumed in the yearly hospital expenditures, and thus are included under the hospitalization costs.
} 
survey was conducted from July 1995 to January 1996. Data were collected for all patients admitted for incomplete abortion in this period. Criteria for inclusion were all patients with incomplete abortion, abortion in progress or a case of dead and retained fetus, who were admitted to the gynecology floor through the emergency ward. One hundred and thirty two patients were included in this survey. The survey excluded cases of dysfunctional bleeding, threatened abortion, and abortions with complications such as uterine perforations and sepsis, and cases that were referred to the hospital from medical offices or other referral sources.

The questionnaire ascertained basic sociodemographic information, surgical procedure received, details of pain management, degree of information provided to patient, level of patient-provider interaction, and details about the family planning counseling session. This questionnaire was supplemented with clinical information obtained from the patient's medical records.

The post-intervention survey of 207 patients was conducted from January to June 1997, using the same instrument.

\section{RESULTS}

\section{Sample Characteristics}

Women in the pre- and post-intervention groups shared similar sociodemographic characteristics. The average age was 26 years in both groups, and nearly $75 \%$ lived permanently with their spouses. Slightly more than three-fifths came from urban areas. Most women were housewives and economically dependent on their partners. ${ }^{6}$

\footnotetext{
${ }^{6}$ Data not shown. Details can be obtained from the authors upon request.
} 
Length of stay

Figure 1 delineates the mean length of patient stay per station in the pre- and postintervention period. Pre-intervention, the average wait was approximately 8.7 hours to undergo D\&C, while post-intervention the mean wait to undergo MVA was 5.8 hours. Once the patient was released from the operative suite, patients waited approximately 17.9 hours to be discharged. This was reduced to 11.1 hours in the post-intervention period. There was not a significant change in the operative time (26.6 minutes for D\&C vs. 30.7 minutes for the MVA procedure). Overall, there was a $35 \%$ reduction in the average total length of stay per patient.

The mean time spent in direct patient care by type of provider in the pre-intervention and post-intervention phases is shown in Table 1. Total pre-surgery (time from admission to D\&C) nursing time decreased by nearly 30 minutes (92.82 minutes pre-intervention vs. 63.84 minutes post-intervention), while physician time decreased by approximately 13 minutes. In the surgical suite, physician time decreased by 24 minutes, while nursing time increased by approximately 10 minutes in the post-intervention period. In the post-surgical recovery phase (D\&C till

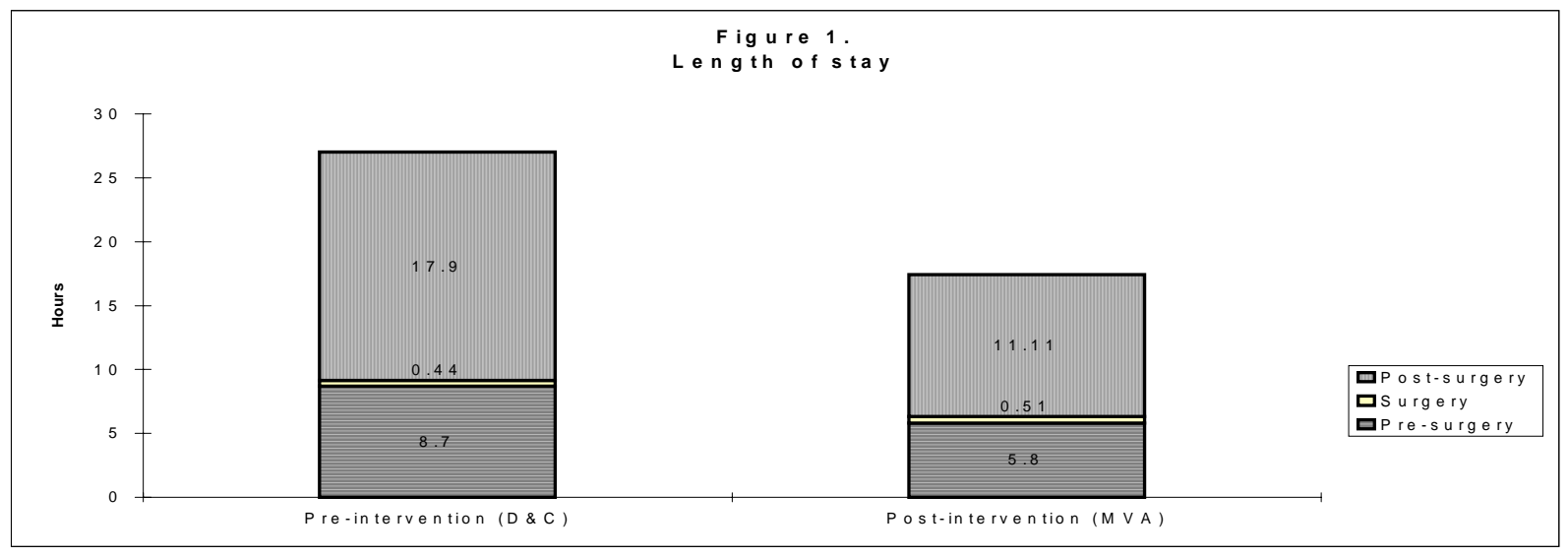


TABLE 1.

MEAN PER-PATIENT TIME OF ATTENTION

BY SERVICE PROVIDER (MINUTES)

\begin{tabular}{|lccccc|}
\hline & \multicolumn{2}{c}{ Nurses } & \multicolumn{2}{c|}{ Doctors } \\
& $\frac{\text { Pre-intervention }}{\underline{(\mathrm{D} \& \mathrm{C})}}$ & $\frac{\text { Post-intervention }}{(\text { MVA })}$ & $\frac{\text { Pre-intervention }}{\underline{(\mathrm{D \& C})}}$ & $\frac{\text { Post-intervention }}{(\text { MVA })}$ \\
Pre-surgery & 92.82 & 63.84 & 88.09 & 75.12 \\
Surgery & 22.18 & 32.12 & 80.27 & 56.16 \\
Post-surgery & 74.82 & 45.12 & 25.55 & 18.16 \\
\hline
\end{tabular}

discharge from hospital), nursing time decreased by nearly 30 minutes; physician time decreased by seven minutes, compared to the pre-intervention period.

\section{Cost of treating patients}

Figure 2 shows the average cost of treating a patient in the pre- intervention and postintervention phases. The average total cost of treating a post-abortion patient in the preintervention phase (using $\mathrm{D} \& \mathrm{C}$ as the procedure of choice) was $\$ 264.47$. This decreased by nearly $32 \%$ to $\$ 180.22$ when the improved service delivery model was implemented. Substantial reductions took place in the categories of hospitalization, staff and instrument $\&$ supply costs, while costs for family planning services increased. As expected, there was substantial intervention cost in the post-intervention period. Table 2 describes these changes in greater detail.

Hospitalization : On a dollar basis, the largest reduction occurred in the costs associated with hospitalization. Pre-intervention hospitalization costs were $\$ 150.77$, and these decreased by nearly $\$ 65$ (43\%) in the post-intervention period to $\$ 85.89$. 


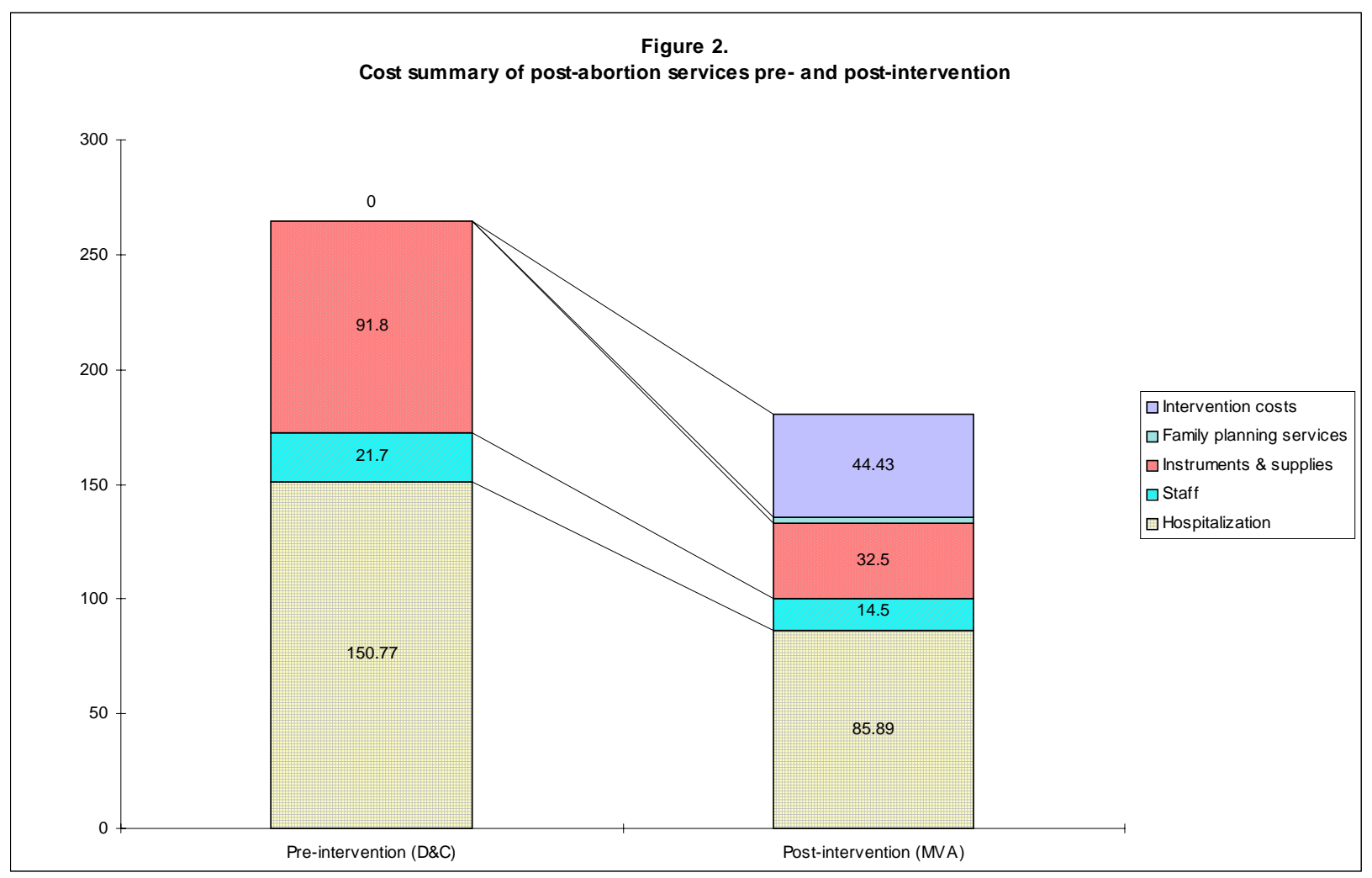

Staff : Staffing costs decreased by $33 \%$, from $\$ 21.70$ in the pre-intervention period to $\$ 14.50$ in the post-intervention period. Nurses constitute the bulk of the personnel costs both in the pre- and post-intervention periods, although there was a reduction in their costs from $\$ 12.70$ to $\$ 9.30$.

Instruments and supplies: Costs of instruments and supplies decreased from $\$ 91.80$ in the preintervention phase to $\$ 32.50$ in the post-intervention phase, a reduction of nearly $65 \%(\$ 59.30)$. Reductions occurred across all categories - instruments (89\%), supplies (52\%), medicines $(68 \%)$ and sterilization materials (60\%). 
TABLE 2.

MEAN PER-PATIENT COSTS OF POST-ABORTION SERVICES PRE- AND POST-INTERVENTION (US DOLLARS, JANUARY 1997)

\begin{tabular}{|c|c|c|c|}
\hline & $\frac{\text { Pre-intervention }}{(\mathrm{D \& C})}$ & $\frac{\text { Post-intervention }}{(\text { MVA })}$ & Change \\
\hline Hospitalization & 150.77 & 85.89 & -64.88 \\
\hline Staff & 21.70 & 14.50 & -7.20 \\
\hline Doctors & 7.60 & 4.80 & -2.80 \\
\hline Nurses & 12.70 & 9.30 & -3.40 \\
\hline Other & 1.40 & 0.40 & -1.00 \\
\hline Instruments \& supplies & 91.80 & 32.50 & -59.30 \\
\hline Instruments & 13.50 & 1.50 & -12.00 \\
\hline Supplies & 34.60 & 16.70 & -17.90 \\
\hline Medicines & 40.70 & 13.10 & -27.60 \\
\hline $\begin{array}{l}\text { Sterilization } \\
\text { materials }\end{array}$ & 3.00 & 1.20 & -1.80 \\
\hline $\begin{array}{l}\text { Family planning } \\
\text { services }\end{array}$ & 0.20 & 2.90 & +2.70 \\
\hline Staff costs & 0.20 & 1.60 & +1.40 \\
\hline Contraceptives & 0 & 1.30 & +1.30 \\
\hline Intervention costs & 0 & 44.43 & +44.43 \\
\hline TOTAL COSTS & 264.47 & 180.22 & - 84.25 \\
\hline
\end{tabular}


Family planning services: Costs of family planning services increased from $\$ 0.20$ to $\$ 2.90$, with increases in both the staff ( $\$ 0.20$ pre-intervention to $\$ 1.60$ post-intervention) and contraceptive costs (zero pre-intervention to $\$ 1.30$ post-intervention).

Intervention costs: The amortized intervention cost per patient was $\$ 44.43$. This represented project costs $(\$ 266,532)$, supplies provided by IPAS $(\$ 3,000)$ and hospital expenses (cost of personnel time for training, follow-up meetings and supervision; valued at \$7,641). ${ }^{7}$

\section{Quality of care}

Results of indicating quality of care are shown in Table 3 . There was no difference between the pre- and post-intervention groups with regards to pain management. Nearly equal percentages of women were given analgesics pre- and post-surgery in the two groups.

There were significant differences in the two groups with regard to information provided to patients, the physician-patient interaction and family planning counseling. Patients were more likely to be informed about their diagnostic results, surgical procedures, and post-operative progress in the post-intervention phase, and patients were more satisfied with the information provided. Physicians were more likely to introduce themselves and address the patient by her name in the post-intervention period, thus increasing the patient's confidence in her treating physician.

Patients were also more likely to receive family planning counseling in the postintervention phase. Providers were more likely to discuss their preferences with them, and

\footnotetext{
${ }^{7}$ Further details can be obtained from the authors. Briefly, the cost $(\$ 277,175)$ was amortized over a 10 year period, and costs for the 15 month intervention period were calculated. These were then divided by the total number of postabortion cases treated in the 15 month period.
} 
patients had a higher likelihood of accepting a method of their choice prior to hospital discharge.

TABLE 3. QUALITY OF POST-ABORTION CARE

\begin{tabular}{|c|c|c|}
\hline & $\frac{\text { Pre-intervention }}{(\mathrm{D} \& C ; n=132)}$ & $\frac{\text { Post-intervention }}{(\mathrm{MVA}: \mathrm{n}=207)}$ \\
\hline \multicolumn{3}{|l|}{ Pain management } \\
\hline received pre-surgery pain treatment & $26.7 \%$ & $25.7 \%$ \\
\hline received post-surgery pain treatment & $97.7 \%$ & $96 \%$ \\
\hline \multicolumn{3}{|l|}{ Information provided } \\
\hline patient was informed about diagnostic result & $68.3 \%$ & $87.3 \% *$ \\
\hline $\begin{array}{l}\text { patient was informed about surgical } \\
\text { procedure }\end{array}$ & $83.2 \%$ & $87.3 \% *$ \\
\hline $\begin{array}{l}\text { patient was informed about post-surgical } \\
\text { progress }\end{array}$ & $19.8 \%$ & $62.6 \% *$ \\
\hline $\begin{array}{l}\text { patient was satisfied with information } \\
\text { received }\end{array}$ & $17.6 \%$ & $72.5 \% *$ \\
\hline \multicolumn{3}{|l|}{ Patient-physician interaction } \\
\hline physician introduced self to patient & $26.1 \%$ & $51 \% *$ \\
\hline physician addressed patient by name & $59.1 \%$ & $84.8 \% *$ \\
\hline patient felt confidence in her physician & $52.4 \%$ & $73.6 \% *$ \\
\hline \multicolumn{3}{|l|}{ Family planning counseling } \\
\hline patient received family planning counseling & $42.4 \%$ & $85.5 \% *$ \\
\hline patient discussed preferred method & $57.1 \%$ & $81.7 \% *$ \\
\hline patient accepted method prior to discharge & $28.8 \%$ & $57 \% *$ \\
\hline
\end{tabular}

$*$ denotes $\mathrm{p}<0.05$ 


\section{DISCUSSION}

The results of this study showed that the improved service delivery model achieved significant cost savings and simultaneously improved quality of care simultaneously for patients undergoing post-abortion treatment. There was a 35\% reduction in the length of stay in the hospital, a figure comparable to that reported in literature. Johnson et al. found that the average duration of stay in Kenyan hospital for patients undergoing D\&C ranged from 40.9 to 100.7 hours, while the average stay for MVA patients ranged from 18.8 to 23.9 hours (Johnson et al., 1993). In the same study, average stay form $D \& C$ patients in Mexico ranged from 11.7 to 29.9 hours, and the stay for MVA patients for the lone hospital performing MVA in their sample was 11.4 hours. In a report from Tanzania, hospital stay for MVA patients was $41 \%$ less for MVA patients than for D\&C patients (Magotti et al., 1995). In this study, Valdivieso Hospital had an average length of stay of 27.04 hours for D\&C patients, and only 17.42 hours for MVA patients, a reduction of nearly $35 \%$.

It is imperative to understand the factors behind this significant reduction, for it is easy to be mislead into thinking that this reduction is due to the introduction of the MVA process alone. While it is incontrovertible that MVA does reduce post-surgical recovery time as the procedure does not use general anaesthesia, reduction in pre-operative waiting time was largely due to the policy of keeping the surgical suites operating 24 hours a day, thus eliminating the wait that these cases had to undergo in the past. The other notable change post-intervention was the increase in the time spent in ultrasound diagnostics. This increased from 8 minutes preintervention to nearly 39 minutes post-intervention, an indication of perhaps the increased attention being paid to improving patient care quality. 
Reporting their results in May 1991 US dollars, Johnson et al. found that the average per-patient cost of D\&C in Mexican hospitals varied from $\$ 79$ to $\$ 235$, and the cost of MVA was $\$ 65.73$, a difference of $17-72 \%$. They reported that hospitalization accounted for the largest proportion of average cost per patient, and personnel costs were the second greatest contributors (Johnson et al., 1993). In our study, the cost (in January 1997 US dollars) of treating patients with D\&C was $\$ 264.47$ and we achieved a 32\% reduction with the introduction of the improved service delivery model to $\$ 180.22$. Assuming an annual case load to 600 post-abortion cases, these costs translate to potential cost savings of $\$ 50,550$ per year.

The bulk of these dollar savings arise from the decreased length of stay in the hospital. Substantial savings were also achieved in reducing the instruments and supplies used in the service delivery model. Reductions were noted across all categories. The cost of supplies decreased because fewer glucose solutions, surgeon gloves and elastic bandages were used in the MVA procedure. While there reduction in medicines was essentially due to decreased use of Xylocaine and Cytotec tablets, there was a significant increase in the use of Teopental. These reflect the different anaesthetic requirements of the MVA procedure as compared to D\&C. The reduction in the cost of instruments was due to the fact that the cost of MVA syringes was considered to be part of the intervention costs, and was subsumed under that category. Studies have shown that the average total patient cost attributable to MVA instruments and resterilization averages 1\% in Mexico and 9-16\% in Kenya (Johnson et al., 1993).

While staffing costs decreased post-intervention, these do not constitute a major bulk of total costs. While nursing costs were more than physician costs, these did not constitute more than $6 \%$ of the total costs, both pre- and post-intervention. This finding was also reported from 
research in Tanzania (Magotti et al, 1995).

As expected, costs for family planning services increased. This was due in equal measure to the time spent by the provider counseling the patient and also in the provision of contraceptives, but overall, cost of these services accounted for only $1.6 \%$ of the total cost.

This study explicitly quantified the costs of intervention, as policy makers need this information to make cost-effectiveness judgements when introducing similar programs in their health care facilities. While IPAS provided supplies free or at low cost (such as training materials, pamphlets, leaflets and posters, etc.) we attempted to quantify these and include them in our calculations. We also included direct project costs (salary of training personnel, training expenses, etc.) and costs of hospital personnel involved in various workshops. Many studies do not take into account of this important cost and do not address it explicitly. We feel it is of utmost importance to discuss these costs up-front, as they constitute a major component $\$ 444.43$ per patient or nearly $25 \%$ ) of the average per- patient cost for the MVA service delivery model.

An important element that this study focuses on is the issue of quality following the implementation of the model. Previous studies have addressed only cost issues, to the total exclusion of quality of care provided. From our data we were able to unequivocally state that quality of care improved following the implementation of the service delivery model. Significant improvements were noted in the patient-physician interaction and information exchange. This engenders greater trust between the provider and the patient, and leads to increased compliance with provider recommendations and advice. This was evidenced from the significantly increased acceptance of family planning methods by patients prior to discharge. 


\section{CONCLUSION}

Our results demonstrate that it is eminently feasible to achieve cost savings without compromising quality of care in post-abortion patients. Future research will demonstrate wether these findings stand the test of time and also if the model is applicable to other facilities in Latin America. This service delivery model can serve as a template for similar programs in other specialities. It must be borne in mind that the success of such a program depends not only on introduction of the improved surgical/medical procedure, but also on concurrent refinement of patient management protocols. Last but not the least, wholehearted support from health facility management and providers is a crucial, but oft neglected, ingredient. 


\section{REFERENCES}

Abernathy M, et al. Manual to Evaluate the Use of Resources for Treatment of Incomplete Abortions, IPAS 1993.

Aggarwal VP, Mati JKG. Epidemiology of Abortion in Nairobi, Kenya. J. Obst. Gynec. E. Cent. Afri. 1: 54, 1982.

Benson RC and Pernoll ML (eds.) 1994. Handbook of Obstetrics and Gynecology, Ninth Edition. McGraw Hill, Inc., Health Professions Division, pp. 288-299.

Careres GH, et al. Manejo Hospitalario del Aborto Incompleto: Estudio Comparativo del Curetaje Utrino versus la Aspiracion por vacio. Corporacion Centro Regional de Poblacion Monografia. 16:45-81, 1981.

Germain A. The Christopher Tietze International Symposium: An Overview. Int. J. Gynaec. Obstet. Suppl 3: 1, 1989.

Johnson BR, Benson J, Bradley J, Ordonez AR. Costs and Resource Utilization for the Treatment of Incomplete Abortion in Kenya and Mexico. Soc Sci. Med. 36:11, 1443-1453, 1993.

La Mujer Adolescente, Adulta, Anciana y su Salud. Secretaria de Salud, Direccion General de Salud Materno Infantil, Programa Nacional "Mujer, Salud y Desarrollo", Mexico, 1992.

Magotti RF, Munjinja PGM, Lema RS, Ngwalle EK. Cost-Effectiveness of Managing Abortions: MVA Compared to Evacuation by Curettage in Tanzania. East Africa Med J. 72:4, 248-251, 1995.

McLaurin KE, et al. Health System Role in Abortion Care: The Need for a Pro-Active Approach: Issues in Abortion Care 1: IPAS, Carrboro, North Carolina, 1991.

von Allmen SD, et al. Costs of Treating Abortion Related Complications. Family Plan. Perspect. 9, 273-276, 1977. 